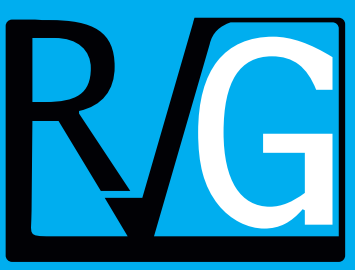

Año 21 No. 74

Abril - Junio 2016

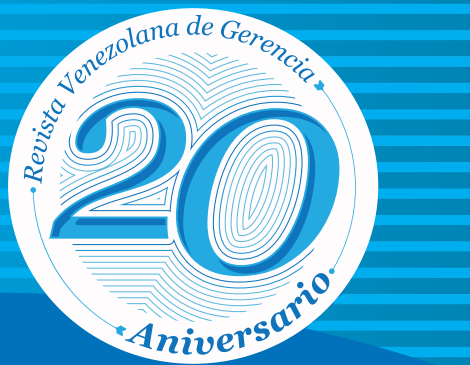

Venezolana de
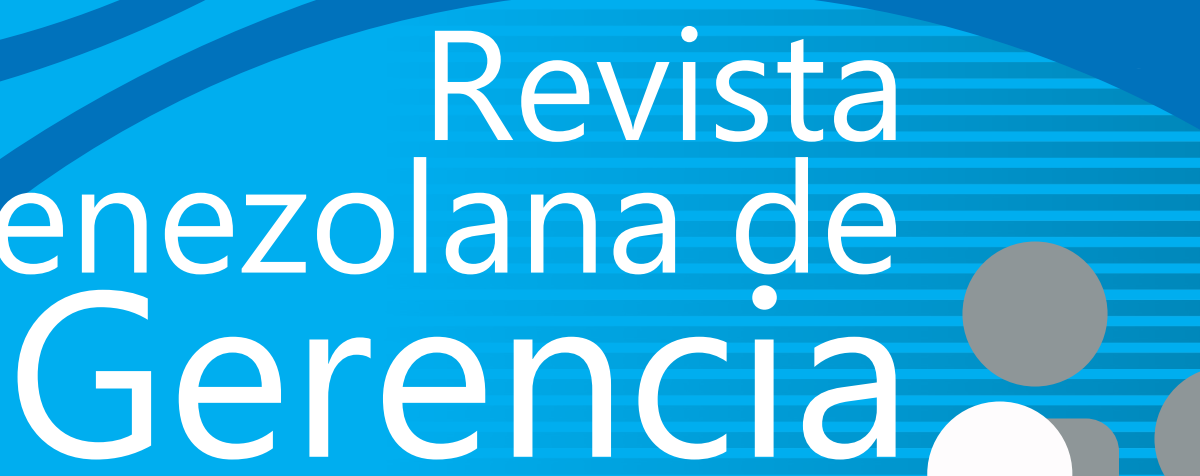


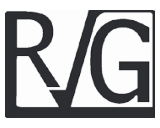

\title{
Características de diseño organizativo de las medianas empresas del estado Lara, Venezuela $^{1}$
}

\author{
Zapata Rotundo, Gerardo J* \\ Sigala Paparella, Luis $\mathrm{E}^{* *}$ \\ Canet Giner, María Teresa***
}

\section{Resumen}

Este trabajo tiene como objetivo describir las características de diseño organizativo de medianas empresas del estado Lara, Venezuela. Para ello, se aborda el estudio en tres niveles de análisis organizacional: micro, meso y macro organizativo, y se lleva a cabo mediante una investigación de carácter cuantitativo. Se caracterizan las medianas empresas según el comportamiento de las variables estructurales centralización, formalización y especialización, y dentro de las formas básicas de organización: orgánica, mecánica y burocrática. Para cumplir con su propósito, se realizó un estudio de campo mediante la aplicación de un cuestionario. Los resultados muestran que el tipo de estructura organizativa de las empresas evaluadas se corresponde en su mayoría a la funcional, y poseen características de formas de organización burocrática y mecánica. La conclusión principal, es que las medianas empresas objeto de estudio tienden a ser formalizadas y centralizadas en su funcionamiento.

Palabras clave: diseño organizativo; estructura organizativa; formas de organización; mediana empresa; variables de diseño organizativo.

Recibido: 20.03.15 Aceptado: 20.03.16

1 Este trabajo es producto del Proyecto Código: 001-AC-2012, financiado por el C.D.C.H.T de la Universidad Centroccidental "Lisandro Alvarado", Venezuela.

* Doctor en Dirección de Empresas por la Universitat de València, España. Profesor Titular del Decanato de Ciencias Económicas y Empresariales de la Universidad Centroccidental "Lisandro Alvarado", Venezuela. E-Mail: zapager@yahoo.com.

** Doctor en Dirección de Empresas, Universitat de València. Profesor Titular del Decanato de Ciencias Económicas y Empresariales de la Universidad Centroccidental "Lisandro Alvarado", Venezuela. E-Mail: Isigala@ucla.edu.ve.

*** Doctora en Ciencias Económicas y Empresariales. Profesora Titular del Departamento de Dirección de Empresas de la Facultad de Economía de la Universitat de València, España. E-Mail: teresa. canet@uv.es. 


\title{
Characteristics of the Organizational Design of Lara Sate's Medium Business, Venezuela
}

\begin{abstract}
This paper aims to describe organizational design characteristics of medium firms from Lara state, Venezuela. With this purpose, the study goes through three levels of organizational analysis: micro, meso and macro levels and is conducted throughout a quantitative research method. Medium firms are characterized according to the behavior of different structural variables like centralization, formalization and specialization, and the basic organizational forms such as organic, mechanic and bureaucratic. To fulfill its purpose, a quantitative research study was conducted using a questionnaire. Among the main results emerged that the type of organizational structure of the evaluated companies mainly corresponds to the functional organizational type and have the characteristics of the bureaucratic and mechanistic forms of organization. The main conclusion is that the medium firms under study tend to be formalized and centralized operation.
\end{abstract}

Key words: organizational design; organizational structure; organizational forms; medium business; variables of organizational design.

\section{Introducción}

El diseño organizativo se refiere al conjunto de actividades, acciones y decisiones estratégicas y operativas que toman directivos y gerentes con el propósito de definir los procesos, la estructura y la forma de organización que adopta la empresa en un momento determinado y bajo ciertas condiciones contextuales (Zapata y Hernández, 2014). De este modo, la presente investigación tiene como objetivo describir las características de diseño organizativo de las medianas empresas del estado Lara, Venezuela. Para ello, se aborda la investigación en tres niveles de análisis organizacional: micro, meso y macro organizativo.

El nivel micro-organizativo, se refiere al estudio de las variables estructurales de diseño organizativo. En ese sentido, se encuentran en la literatura de la teoría de la organización una diversidad de modelos que en su mayoría coinciden en su conceptualización y aplicación práctica (Miller, 1987; Mintzberg, 1999; Daft, 2000; Donaldson, 2001; Prakash y Gupta, 2008; Glock y Broens, 2013; Hwang y Norton, 2014; MarínIdárraga y Losada-Campos, 2015), e incluyen algunas de las siguientes variables: centralización, formalización, complejidad, especialización, intensidad administrativa, sistemas de incentivos $y$ puestos de trabajos.

Por su parte, el nivel mesoorganizativo está vinculado con la definición de la estructura organizativa de la empresa, y referido a cuatro tipos básicos: (a) simple; (b) funcional; (c) divisional; y (d) matricial. Finalmente, el nivel macro-organizativo es el resultado de la gestión de las variables de diseño incorporadas en los niveles micro y meso organizativo, que, según su comportamiento, determinarán si la empresa se sitúa dentro del marco de la teoría orgánica -forma orgánica o mecánica- (Zanzi, 1987; Courtright et al., 1989; Russell y Russell, 1992; 
Características de diseño organizativo de las medianas empresas del estado Lara...

Gerardo J. Zapata Rotundo, Luis E. Sigala Paparella, María Teresa Canet Giner

Donaldson, 2001; García, 2003; Claver et al., 2006; Claver et al., 2012), o de la teoría burocrática -forma burocrática simple o compleja- (Mansfield; 1973; Child, 1973; Grinyer y Ardekani, 1981; Donaldson, 2001; Prakash y Gupta, 2008; Mao et al., 2009; Zapata y Hernández, 2014).

Cabe destacar que el estudio de las pequeñas y medianas empresas (Pymes) ha alcanzado un lugar de mucha importancia en el ámbito académico y empresarial por tener un impacto muy significativo en la evolución y desarrollo de la estructura productiva de la economía global, constituyéndose en un importante proveedor de empleos y desarrollo de nuevos productos y mercados (Aragón y Rubio, 2009; Boonpattarakan, 2012; López et al., 2012; Aristy, 2012; Gallardo y Avilés 2014; Arteaga et al., 2014; Tyulkova, 2014). En el caso Venezolano, y de acuerdo al IV Censo Económico (20072008) realizado por el Instituto Nacional de Estadística (INE,2010), el 98\% de las empresas venezolanas son Pymes; sin embargo, las investigaciones relacionadas con las características de su diseño organizativo, en especial en lo referido al estudio de los tipos de estructuras organizativas y las formas de organización que adoptan, no han sido abordadas con tanta amplitud en la literatura de la teoría de la organización en Venezuela como sí en otros escenarios.

La metodología de investigación utilizada para alcanzar el objetivo planteado en el presente trabajo, es de naturaleza cuantitativa. De este modo, las unidades de análisis la conformaron una población de 411 medianas empresas de todos los sectores de la economía del estado Lara, Venezuela. Dicha población, se organizó a partir de las bases datos de la Cámaras de Comercio e Industriales del Estado Lara, Venezuela.

Se escoge dentro de las empresas Pymes, solo el grupo de las medianas empresas para prescindir de aquellas con estructuras muy simples y poco elaboradas a fin de tener una población lo más homogénea posible que permitiera lograr una mejor identificación de sus características de diseño organizativo. Se tomó como referencia el criterio del Instituto Nacional de Estadística de Venezuela (INE, 2010), que clasifica a la mediana empresa como aquella que ocupa entre 21 a 100 trabajadores. La muestra de estudio la conformaron finalmente 61 medianas empresas que respondieron válidamente los cuestionarios.

Se diseñó el cuestionario para obtener información sobre el sector económico, la edad, el tamaño, el tipo de estructura, y el ámbito de acción de las empresas objeto de estudio, que además contiene las escalas para medir las variables de diseño: centralización, formalización y especialización, utilizándose un escalamiento tipo Likert del 1 al $5^{2}$. Con los datos obtenidos en el trabajo de campo, se procedió a evaluar la fiabilidad de dichas escalas a través del modelo de consistencia interna alpha de Cronbach (1951), cuyos resultados arrojaron para la escala de medición de la variable centralización un valor de 0,873 , para la de la formalización de 0,900 , y de 0,688 en el caso de la escala

2 Escalamiento: muy desacuerdo $=1$, en desacuerdo $=2$, a veces $=3$, de acuerdo $=4$, muy de acuerdo $=5$. 
de la especialización. Valores superiores o muy próximos al mínimo de 0,700 recomendado por la literatura (Nunally y Bernstein, 1995; Hair et al., 1999).

Los cuestionarios fueron enviados a la población vía correo electrónico, y en algunos casos se aplicaron a través de entrevistas personales; estaban dirigidos a cargos directivos o de la alta gerencia. El envío se hizo en dos oleadas y con intervalo de tres meses, la primera en octubre de 2013 y la segunda en enero de 2014. El tratamiento de los datos se llevó a cabo con el apoyo del programa estadístico SPSS 18.0.

\section{Nivel micro-organizativo: las variables de diseño organizativo}

El estudio analiza básicamente las variables: centralización, formalización y especialización, puesto que son consideradas en los modelos de dimensiones o variables de diseño como las más significativas en la definición de las características de las estructuras y formas de organización que adoptan las empresas (Child, 1973; Miller, 1987; Mintzberg, 1999; Daft, 2000; Donaldson, 2001; Prakash y Gupta, 2008; Glock y Broens, 2013; Delić y Ahmetović, 2013; Marín-Idárraga y Losada-Campos, 2015).

\subsection{Centralización}

En la definición clásica, se asocia a la centralización con la ubicación y la distribución de la autoridad para la toma de decisiones entre los diferentes componentes de la estructura organizativa que afectan a toda la organización y que puede ser delegada a través de ella (Pugh y Hickson, 1976).
Se corresponde a la asignación y localización en la estructura organizativa el derecho, la autoridad, el poder para tomar decisiones y la distribución de recursos (Glock y Broens, 2013; Reichmann y Rohlfing, 2014, Foss et al., 2015).

Así, y en términos generales, cuando la toma de decisiones es centralizada ésta recae en la alta dirección o en los niveles superiores de la jerarquía empresarial. Al contrario, es descentralizada cuando parte de la toma de decisiones vinculada con el trabajo operativo y estratégico es delegada y llevada a cabo de manera más discrecional por los gerentes y empleados de los niveles jerárquicos medios e inferiores (Pertusa et al., 2010; Hempel et al., 2012; Delić y Ahmetović, 2013; Wynen et al., 2014; Reichmann y Rohlfing, 2014; Zapata y Hernández, 2014; Marín-Idárraga y Losada-Campos, 2015).

Cabe destacar que la centralización/descentralización puede estar influenciada por los límites específicos de cada individuo y su formación, el nivel de riesgo aceptado por el subordinado para decidir, la complejidad del trabajo y el estilo de liderazgo. Con lo cual, el comportamiento de la variable centralización no debe observarse en términos absolutos, sino más bien como un continuum donde existen grados diferentes de centralización o descentralización.

\subsection{Formalización}

La formalización constituye el grado en el cual la organización depende de reglas, instrucciones, procedimientos escritos y estandarizados que predeterminan el comportamiento, las 
Características de diseño organizativo de las medianas empresas del estado Lara...

Gerardo J. Zapata Rotundo, Luis E. Sigala Paparella, María Teresa Canet Giner

comunicaciones y las tareas de sus miembros (Pleshko, 2007; Prakash y Gupta, 2008; Delić y Ahmetović, 2013; Marín-Idárraga y Losada-Campos, 2015).Con lo cual, se reconoce por ser explícita y codificada (Nasurdin et al., 2006; Fiedler y Welpe, 2010; Hempel et al., 2012; Glock y Broens, 2013; Foss et al., 2015) y se identifica en la literatura de teoría de la organización, al igual que la centralización, como una forma de establecer mecanismos de control y coordinación de las actividades en la empresa (Nohria y Ghoshal, 1994; Hodge et al., 1998; Bodewes, 2002; González, et al., 2012).

Asimismo, la formalización ayuda a reducir la incertidumbre, la ambigüedad - la interpretación inadecuada de las tareas que deben realizar los empleados (Bonner et al., 2002; Demirci, 2013; Zapata y Hernández, 2014). Con ella, se busca proporcionar una adecuada garantía para que se cumpla con el trabajo en la organización, entendiéndose que si está altamente formalizado entonces el empleado tendrá poco discrecionalidad en la manera de llevarlo a cabo (Liao, 2007; Zapata y Canet, 2012; Glock y Broens, 2013).

\subsection{Especialización}

$$
\text { El estudio clásico de Pugh }
$$
y Hickson (1976) se refiere a la especialización como el nivel de separación de las actividades llevadas a cabo por los individuos en las unidades organizacionales, lo que implica una mayor especificidad y una reducción del número de tareas asignadas. Se refiere entonces al desglose de las metas de la organización en diferentes tareas, subtareas o habilidades específicas que se refleja en toda la cadena de valor (Fiedler y Welpe, 2010; Puranam et al., 2014). Tiene que ver con el principio de división del trabajo, el cual postula que el trabajo en la organización para ser más eficiente debe repartirse entre una gran cantidad de individuos, por lo que cada uno será responsable de una pequeña cantidad de tareas correspondientes a todo el proceso de trabajo en general (Hall, 1996; Zapata y Canet, 2012; Glock y Broens, 2013).

\section{Nivel meso-organizativo: la estructura organizativa}

El análisis del diseño de la estructura organizativa se ubica dentro del marco de la teoría contingente. Esta teoría parte de la idea de que no existe una manera única de diseñar a la organización, ya que plantea la presencia de una serie de factores contextuales, 0 de contingencia ${ }^{3}$, que determinan la naturaleza de sus procesos y de su estructura organizativa. Por tanto, es necesario el ajuste de las variables organizacionales con esos condicionantes contextuales para que la empresa alcance un desempeño superior (Donaldson, 2001; Simon, 2007; Liang et al., 2010; Pertusa, et al., 2010; Zapata y Mirabal; 2013; Zainun et al., 2014), de este modo, las organizaciones que logren una mejor adaptación y ajuste con esos factores de contingencia, serán las más eficientes y tendrán, por tanto, una mayor probabilidad de supervivencia (Donalson, 2001; Volberda et al., 2012; Akingbola, 2013; Kloviene, 2013).

3 Algunos factores de contingencia son: el entorno, la edad, el marco institucional y el tamaño de la organización, la tecnología, el conocimiento y la estrategia organizacional. 
El concepto de estructura organizativa ha sido abordado por los investigadores en el campo de la teoría de las organizaciones bajo diferentes definiciones. Sin embargo, en la gran mayoría de los casos coinciden en que constituye una red de unidades entrelazadas por un sistema de relaciones formales de autoridadsubordinación, donde confluyen una serie de actividades estandarizadas y coordinadas bajo una serie de normas y reglas de comportamiento preestablecidas que en su conjunto buscan contribuir al logro de los objetivos de la empresa como un todo.

Se considera a la estructura organizativa como el arreglo de las partes de la organización, en los cuales se incluyen elementos tales como: la agrupación de las actividades y líneas de comunicación formal, el tamaño de las unidades, la coordinación y el control jerárquico, el grado de formalización y normalización del comportamiento de los miembros de la organización, y, además, muestra hasta qué punto se aprueba y se pone en marcha el grado de autoridad para la toma de decisiones (Hall, 1996; Hodge et al., 1998; Donalson, 2001; Chen et al., 2010; Zapata y Hernández, 2014).

De este modo, la estructura puede observarse como un recurso o como una capacidad de la organización que contribuye al desarrollo e implementación de las estrategias necesarias para alcanzar ventajas competitivas (Pertusa et al., 2010; Claver et al., 2012), convirtiéndose, por tanto, en un determinante de su desempeño (Donalson, 2001; Csaszar, 2012; Volberda et al., 2012; Claver et al., 2012; MarínIdárraga y Cuartas-Marín, 2013), se constituye es un medio para difundir, gestionar y almacenar el conocimiento por toda la organización (Nonaka y Takeuchi, 1995; Fiedler y Welpe, 2010; Mahmood et al., 2013; Salim y Abdalraheem, 2013; Tyulkova, 2014), y tiene un vínculo muy importante con la cultura organizacional (Janićijević, 2013).

La estructura organizativa responde así a la naturaleza de la organización y a sus propias necesidades, al comportamiento de sus variables de diseño y a la particular influencia que sobre ella ejercen los factores contextuales, haciéndose intransferible y poco imitable para otras organizaciones, siendo, por tanto, el resultado de una acción deliberada y racional (Donalson, 2001; Biggiero y Laise, 2003; Tiller, 2012).

Toman cuatro formas básicas: funcional/simple, funcional/compleja, divisional y matricial, aunque la literatura más reciente viene señalando la existencia de nuevas formas organizativas como la estructura hipertexto de Nonaka y Takeuchi (1995), la organización virtual, la organización sin límites o la organización que aprende (Child, 2008; Salazar, 2005), y la estructura en redes de cooperación (Narváez y Fernández, 2013; Pelechano et al., 2015) que le proporcionan a las organizaciones involucradas en ellas la agilidad y la flexibilidad necesaria para enfrentar los entornos cambiantes y complejos, así como un intercambio y complementariedad de recursos y capacidades.

La estructura funcional/simple es el modelo más sencillo, especialmente adecuado para pequeñas empresas con pocos niveles jerárquicos, tal vez a lo sumo tres o cuatro, donde el primero corresponde a los propietarios o directivos y los siguientes a la base operativa que lleva a cabo el trabajo técnico y operativo, sin que haya una fuerte división del trabajo $\mathrm{y}$, por ende, un bajo valor para la variable de diseño 
Características de diseño organizativo de las medianas empresas del estado Lara...

Gerardo J. Zapata Rotundo, Luis E. Sigala Paparella, María Teresa Canet Giner

especialización. Asimismo, puede observarse en este tipo de estructura altos niveles de centralización y bajos de formalización.

Por su parte, la estructura funcional/compleja, que al igual que la funcional/simple, reúne en unidades orgánicas $^{4}$ específicas a personas 0 cargos que realizan actividades similares; es decir, se integran en unidades con funciones homogéneas, por ejemplo: administración, ventas, producción, almacén, distribución, producción, compras, recursos humanos, calidad, entre otras. La diferencia fundamental con la estructura funcional/simple radica en el hecho de que posee un mayor número de niveles de jerarquías y de unidades orgánicas; es decir, una mayor diferenciación vertical y horizontal (Daft, 2000; Zapata y Hernández, 2014).

En la divisional, las unidades orgánicas que la definen como "divisional" se forman a partir de los criterios de agrupación por productosservicios, clientes o áreas geográficas, y cuya denominación con frecuencia es la "división". Surge, fundamentalmente, cuando la incorporación de nuevas unidades en una empresa se torna demasiado compleja en respuesta a la necesidad de comercializar o elaborar diversos productos poco relacionados o muy diferenciados tecnológica y comercialmente, lo cual hace difícil su administración y coordinación bajo una estructura de naturaleza funcional (Daft, 2000; Gibson et al., 2011; Delić y Ahmetović, 2013; Zapata y Hernández, 2014).

Por su parte, la estructura matricial trata de hacer más flexibles las estructuras tradicionales al formar equipos de proyectos interdisciplinarios cuando se requiere llevar a cabo la producción de múltiples bienes o servicios con ciclo de elaboración y permanencia limitadas en el tiempo o a corto plazo. Se basa entonces en la existencia al mismo tiempo, y de manera solapada, de agrupaciones funcionales a través de unidades de línea que forman las columnas o dimensión vertical de la matriz y la agrupación por proyectos alrededor de un producto o servicio que constituye las filas o la dimensión horizontal de esa matriz (Hodge et al., 1998; Daft, 2000; Gibson et al., 2011; Zapata y Hernández, 2014). En resumen, es un diseño estructural donde se asignan recursos de las unidades de línea a los proyectos que se forman.

\section{Nivel macro-organizativo: las formas de organización}

Son el resultado de las decisiones y la gestión de las variables de diseño incorporadas en los niveles micro y meso organizativo, que situarán a la empresa bien sea en el marco de la teoría orgánica: forma orgánica $o$ mecánica, o de la teoría burocrática: forma burocrática simple o compleja.

Burns y Stalker desarrollaron una teoría de la estructura organizativa denominada teoría orgánica, cuyas características se consolidaron con las contribuciones de trabajos posteriores (Milne, 1970; Zanzi, 1987; Courtright et al., 1989; Russell y Russell, 1992; Donaldson, 2001; García, 2003; Claver et al., 2006; Ramezan, 2011; Claver et al., 2012).

4 Por ejemplo: Asamblea de Accionistas, Junta Directiva, Gerencia de Operaciones, Gerencia General, Gerencias Medias, Departamentos, Secciones y Coordinaciones. 
En ella, se identificó la forma mecánica con un parecido al tipo de modelo burocrático ideado por Weber (1947), y la forma orgánica cuyas características son opuestas: flexibles estructural y operativamente, y muy adaptables a las condiciones cambiantes.

Así, la forma mecánica se caracteriza por: (a) una descripción precisa y documentada de las tareas de cada cargo, lo cual define una alta formalización; (b) una estructura de supervisión y control donde las decisiones se toman principalmente en la jerarquía superior de la pirámide empresarial, lo que denota una importante centralización; y (c) una alta separación del trabajo que se traduce en elevada diferenciación estructural -vertical y horizontal-, especifica una alta especialización.

Por su parte, en la forma orgánica resaltan las siguientes propiedades: (a) el ajuste y la redefinición continua de las tareas y el alto compromiso hacia el trabajo, produce una tendencia a una baja formalización; (b) la estructura informal de control y autoridad, la participación de los individuos en la toma de decisiones y la comunicación lateral sugiere una tendencia hacia una menor centralización; y (c) la localización del conocimiento en toda la red organizacional y el hecho de que los individuos puedan trabajar simultáneamente con diferentes capacidades, define una baja especialización.

Dentro del marco de la teoría burocrática, se desprende la idea de que la forma de organización se desarrolla, al igual que en la teoría orgánica, en un continuum que se mueve desde un extremo con una forma burocrática simple y en el otro extremo con una más compleja estructuralmente. Esta teoría ve a la centralización negativamente relacionada con la especialización y la formalización (Mansfield; 1973; Child, 1973; Grinyer y Ardekani, 1981; Donaldson, 2001; Prakash y Gupta, 2008; Mao et al., 2009).

De este modo, y siguiendo los planteamientos de esos autores, la burocracia cuando se descentraliza adquiere un mayor grado de formalización como mecanismo alterno de control que suple a la supervisión directa de los trabajadores. Se observa en un extremo del continuum a la estructura simple. En ella, la coordinación se ejecuta a través de la supervisión directa, donde el poder de decisión está en manos de un directivo o de un gerente general ocasionando una alta centralización en la toma de decisiones; a su vez, el comportamiento está poco formalizado, lo cual significa una escasa descripción de las tareas y procedimientos de trabajo en documentos o manuales.

En el otro extremo del continuum burocrático, existen formas de organización más complejas y descentralizadas, que, a su vez, presentan alta formalización y especialización debido a que se le da mayor énfasis a la división del trabajo. Es decir, se está frente a un tipo de organización unida por normas, reglamentos, jerarquía de autoridad, rutinas y procedimientos estandarizados con el propósito principal de controlar el comportamiento y desempeño de sus miembros.

Tras esta revisión teórica, y en función de los objetivos fijados en la introducción de este trabajo, se pueden plantear un conjunto de hipótesis que el análisis empírico intentará contrastar.

H1: Las formas de organización orgánica o mecánica se definen del siguiente modo a través de las variables centralización, formalización y especialización: 
Características de diseño organizativo de las medianas empresas del estado Lara...

Gerardo J. Zapata Rotundo, Luis E. Sigala Paparella, María Teresa Canet Giner

H1.a: En la forma de organización mecánica, las variables de diseño centralización, formalización y especialización tienden a ser elevadas.

H1.b: En la forma de organización orgánica, las variables de diseño centralización, formalización y especialización tienden a adoptar valores bajos.

H2: Las formas de organización burocrática, simples o complejas, se definen del siguiente modo a través de las variables centralización, formalización y especialización:

H2.a: En la burocracia-simple, hay una tendencia a una elevada centralización acompañada de baja formalización y especialización

H2.b: En la burocracia compleja, la tendencia es hacia la descentralización junto a elevados niveles de formalización $y$ especialización que permitan mantener el control.

H3: En el marco de la teoría burocrática, existe una relación inversa entre la variable centralización y las variables formalización y especialización.

\section{Diseño organizativo en las medianas empresas del Estado Lara, Venezuela}

En esta sección del trabajo, se procede a evaluar las características de diseño organizativo de las medianas empresas del estado Lara, Venezuela. Para ello, se abordan los tres niveles de análisis desarrollados previamente en el marco teórico: (a) micro-organizativo; (b) mesoorganizativo; y (c) macro-organizativo. Cabe destacar que el análisis del nivel micro y macro organizativo se realizó en conjunto, puesto que dependiendo del comportamiento de las variables de diseño se define la forma de organización que adopta la empresa.

\subsection{Tipo de estructura organizativa}

Para identificar el tipo de estructura en el nivel meso-organizativo, se llevó a cabo un análisis estadístico descriptivo a través de una tabla de contingencia partiendo de los datos obtenidos de la primera parte del cuestionario (datos de identificación). En la tabla 1 se ofrecen los resultados.

Tabla 1

Tipos de Estructuras Organizativas

\begin{tabular}{cccccccccc}
\hline $\begin{array}{c}\text { Niveles }^{1} \\
\text { Jerárquicos }\end{array}$ & \multicolumn{3}{c}{ Funcional } & & Divisional & $\begin{array}{c}\text { Total de } \\
\text { Empresas }\end{array}$ & $\begin{array}{c}\text { Nro. de } \\
\text { Unidades } \\
\text { Orgánicas }\end{array}$ \\
& Funcional/Simple & Funcional/Compleja & & & & & \\
\hline & Cantidad & $\%$ & Cantidad & $\%$ & Cantidad & $\%$ & Cantidad & $\%$ & \\
\hline 4 & 4 & 6,56 & 0 & 0 & 0 & 0,00 & 4 & 6,56 & Hasta 6 \\
5 & 0 & 0,00 & 24 & 39,34 & 0 & 0,00 & 24 & 39,34 & De 6 a 11 \\
6 & 0 & 0,00 & 21 & 34,43 & 0 & 0,00 & 21 & 34,43 & De 9-15 \\
7 & 0 & 0,00 & 10 & 16,39 & 2 & 3,28 & 12 & 19,67 & De 11-15 \\
Totales: & 4 & 6,56 & 55 & 90,16 & 2 & 3,28 & 61 & 100 & \\
\hline
\end{tabular}

Fuente: Elaboración propia. 
Se observa en la tabla 1 que el $96,72 \%$ de las empresas (la suma de la estructura funcional/simple y la funcional/compleja), tienen estructuras organizativas funcionales, y soloel $3,28 \%$, son divisionales. De las estructuras funcionales, el 6,56\%, 4 empresas del total de la muestra, son del tipo funcional/ simple puesto que poseen hasta 4 niveles de jerarquía que se corresponde a una de sus características principales. Concentrándose la mayoría de empresas, el 90,16\%, como estructuras funcional/compleja puesto que tienen entre 5 y 7 niveles de jerarquía, lo cual refleja una característica estructural importante de la población de medianas empresas estudiadas. Notándose también que en la medida que se pasa de una estructura funcional/simple a una funcional/compleja o divisional, se observa que hay un aumento tanto en el número de niveles jerárquicos como de unidades orgánicas, situación que se corresponde con las características de estos tipos de estructura organizativas, tal como lo señala la teoría.

Por otra parte, la baja presencia en los resultados del estudio de organizaciones con estructuras divisional, refleja el hecho de que las medianas empresas se dedican básicamente a producir o comercializar un solo tipo de producto, o en caso de que lo hagan con varios estos deben estar muy relacionados tecnológica y comercialmente. Esta es una característica principal de la estructura funcional y una de las principales diferencias con la divisional.

Por último, no se ha identificado el modelo de organización matricial, quizás por tratarse de empresas medianas con una orientación más tradicional en cuanto a la dinámica de los productos que elaboran y comercializan; es decir, con una permanencia prolongada $y$ estable en el tiempo, con procesos de innovación poco intensivos y que no trabajan bajo el concepto de proyectos tal y como se concibe en el tipo de estructuras matriciales.

\subsection{Comportamiento de las variables de diseño en la definición de las formas de organización}

Paradeterminarlascaracterísticas de diseño organizativo relacionado con el nivel macro-organizativo referido a la determinación de la forma de organización mecánica, orgánica o burocrática -simple o compleja- que adoptan las población de medianas empresas estudiadas, se aplicó en el tratamiento de los datos los siguientes métodos estadísticos multivariantes: análisis de conglomerados jerárquico y $\mathrm{K}$-medias, ANOVA de un factor y análisis discriminante.

El análisis de conglomerados jerárquico proporcionó, a través del dendograma, el número de formas de organización. De este modo, se utilizó como variables de agrupación los ítems de las escalas para medir las variables centralización, formalización y especialización (nivel microorganizativo), aplicando el método de conglomeración de Ward y la medida de distancia de euclídea al cuadrado. Después de su análisis, se decidió que la solución natural para este caso era una clasificación de tres grupos de empresas (G1, G2 y G3).

Para validar la clasificación obtenida y asegurar que se refleja la estructura subyacente de la población objeto de estudio, se realizó un análisis discriminante con el propósito de 
Características de diseño organizativo de las medianas empresas del estado Lara... Gerardo J. Zapata Rotundo, Luis E. Sigala Paparella, María Teresa Canet Giner

presentar la matriz de confusión. Esta, ofrece los resultados de la bondad de la clasificación al cruzar la predicción de pertinencia de acuerdo a la función discriminante, con la ubicación de las empresas en cada grupo según lo obtenido del análisis de conglomerado (cluster). Los resultados en la tabla 2.

\section{Tabla 2}

\section{Matriz de Confusión del Análisis Discriminante}

\begin{tabular}{llccccc}
\multicolumn{2}{c}{ Clasificación del Cluster } & \multicolumn{3}{c}{ Grupo de Pertenencia Pronosticado } & Total \\
& & $\mathbf{G 1}(\mathbf{N E = 1 0 )}$ & $\mathbf{G 2}$ (NE=29) & $\mathbf{G 3}$ (NE=22) & Cluster \\
\hline Burocracia Compleja & $(\mathrm{G} 1)$ & $\mathbf{1 0}(\mathbf{1 0 0 \% )}$ & 0 & 0 & 10 \\
Mecánica & $(\mathrm{G} 2)$ & 0 & $\mathbf{2 8}(\mathbf{9 6 , 6 \% )}$ & 1 & 29 \\
Burocracia Simple & $(\mathrm{G} 3)$ & 0 & 0 & $\mathbf{2 1}(\mathbf{9 5 , 5 0 \% )}$ & 22 \\
\hline
\end{tabular}

Nota: Clasificados correctamente el $\mathbf{9 6 , 7 0 \%}$ de los casos originalmente agrupados.

$\mathrm{NE}=$ Cantidad de empresas por grupo según el análisis de conglomerado.

Fuente: Elaboración propia.

Se observa que la clasificación realizada a través de ambas técnicas estadísticas se corresponden claramente, puesto que hay una tasa de coincidencia bastante elevada del $96,70 \%$, lo que indica una correcta clasificación en tres grupos de formas de organización generada desde el análisis de conglomerados jerárquico.

Por su parte, el análisis de conglomerados de K-medias permitió obtener los valores de la media de cada variable de diseño evaluado en los grupos de forma de organización. Esta última prueba, fue acompañada con un análisis de varianza de un factor para determinar si la media poblacional de las variables dependientes -centralización, formalización y especialización- en cada nivel de la variable independiente -grupos de clasificación: G1, G2 y G3- difieren significativamente. Así, los resultados obtenidos al aplicar e interpretar estas pruebas estadísticas se presentan en la tabla 3 .

Tabla 3

\section{Medias de las Variables de Diseño en cada Forma de Organización}

\begin{tabular}{cccccc}
$\begin{array}{c}\text { Variables de } \\
\text { Diseño } \\
\text { Organizativo }\end{array}$ & $\begin{array}{c}\text { Grupo 1 (G1) } \\
\mathrm{NE}=10(16,39 \%)\end{array}$ & $\begin{array}{c}\text { Grupo 2 (G2) } \\
\mathrm{NE}=29(47,54 \%)\end{array}$ & $\begin{array}{c}\text { Grupo3 (G3) } \\
\mathrm{NE}=22(36,07 \%)\end{array}$ & $\begin{array}{c}\text { ANOVA } \\
\text { Significatividad }\end{array}$ & $\begin{array}{c}\text { Estadístico de Levene } \\
\text { Significatividad }\end{array}$ \\
\hline Centralización & 2,93 & 4,13 & 4,14 & $\mathrm{~F}=27,64 \mathrm{Sig}=0,000$ & $\mathrm{EL}=1,493 \mathrm{Sig}=0,233$ \\
\hline Formalización & 4,21 & 4,34 & 3,01 & $\mathrm{~F}=65,87 \mathrm{Sig}=0,000$ & $\mathrm{EL}=1,982 \mathrm{Sig}=0,147$ \\
\hline Especialización & 3,65 & 3,98 & 3,27 & $\mathrm{~F}=13,35 \mathrm{Sig}=0,000$ & $\mathrm{EL}=1,894 \mathrm{Sig}=0,160$ \\
\hline
\end{tabular}

$\mathrm{NE}=$ representa el número de empresas por cada grupo formado.

Fuente: Elaboración propia. 
Se aprecia en la tabla 3, que el análisis de la varianza arrojó que la media de cada grupo o tipo de forma organizativa (G1, G2 y G3) son diferentes significativamente en cada variable de diseño organizativo, puesto que el valor de la significatividad para el indicador $\mathrm{F}$ en todo los casos es 0,000 (significativa al nivel 0,01). Asimismo, el test de Levene dio como resultado una significatividad superior al $5 \%$ (no significativo) en cada una de las variables dependiente, por lo que se acepta la hipótesis nula $(\mathrm{H} 0)$ de homoscedasticidad que establece que las varianzas son iguales en todos los grupos $y$, por tanto, los resultados obtenidos del ANOVA son consistentes.
Ahora bien, el ANOVA de un Factor señala que la media de las variables de diseño difieren en los grupos de empresas, pero esto no quiere decir que todas sean distintas entre sí estadísticamente. En ese sentido, se aplicó la prueba HSD de Tukey de comparación múltiple en el análisis de la varianza para determinar exactamente donde se ubicaban tales diferencias, cuyos resultados se ofrecen en la tabla 4. Cuestión que era necesaria realizar para caracterizar a las empresas con la mayor precisión posible dentro del grupo de forma organizativa a la cual pertenecen.

\section{Tabla 4}

\section{Prueba HSD de Tukey (Comparación Múltiple)}

\begin{tabular}{|c|c|c|c|c|c|}
\hline Variable Dependiente & $\begin{array}{l}\text { Grupo } \\
\text { (I) }\end{array}$ & $\underset{(J)}{\text { Grupo }}$ & $\begin{array}{l}\text { Diferencia de medias } \\
(I-J)\end{array}$ & Error típico & Significativo \\
\hline \multirow[t]{6}{*}{ CENTRALIZACION } & 1,00 & 2,00 & $-1,20862\left(^{*}\right)$ & , 17287 & ,000 \\
\hline & & 3,00 & $-1,21705\left(^{*}\right)$ & 17978 & 000 \\
\hline & 2,00 & 1,00 & $1,20862\left(^{*}\right)$ & , 17287 & ,000 \\
\hline & & 3,00 &,- 00842 & 13328 & ,998 \\
\hline & 3,00 & 1,00 & $1,21705\left(^{*}\right)$ & , 17978 &, 000 \\
\hline & & 2,00 & ,00842 & , 13328 & ,998 \\
\hline \multirow[t]{6}{*}{ FORMALIZACION } & 1,00 & 2,00 &,- 12562 & , 15630 & ,702 \\
\hline & & 3,00 & $1,20779\left(^{*}\right)$ & , 16255 & 000 \\
\hline & 2,00 & 1,00 & , 12562 & , 15630 & ,702 \\
\hline & & 3,00 & $1,33341\left(^{*}\right)$ & 12051 &, 000 \\
\hline & 3,00 & 1,00 & $-1,20779\left(^{*}\right)$ & , 16255 &, 000 \\
\hline & & 2,00 & $-1,33341\left(^{*}\right)$ & 12051 & 000 \\
\hline \multirow[t]{6}{*}{ ESPECIALIZACION } & 1,00 & 2,00 &,- 32701 & ,17867 & ,169 \\
\hline & & 3,00 & ,38485 (*) & , 18582 &, 00 \\
\hline & 2,00 & 1,00 & ,32701 & ,17867 & , 169 \\
\hline & & 3,00 &, $71186\left(^{*}\right)$ & 13775 & ,000 \\
\hline & 3,00 & 1,00 &,$- 38485\left(^{*}\right)$ & , 18582 &, 000 \\
\hline & & 2,00 &,$- 71186\left(^{*}\right)$ & 13775 & 000 \\
\hline
\end{tabular}

Nota: ${ }^{*}=$ La diferencia de las medias es significativa a nivel 0,05 .

Fuente: Elaboración propia. 
Características de diseño organizativo de las medianas empresas del estado Lara...

Gerardo J. Zapata Rotundo, Luis E. Sigala Paparella, María Teresa Canet Giner

De acuerdo a los resultados derivados del test de Tukey y presentados en la tabla 4, se observa que la variable centralización en el grupo uno (G1) difiere significativamente de las medias de los grupos dos y tres ( $G 2$ y G3), pero en estos dos grupos sus medias no son distintas estadísticamente aunque sus valores cuantitativamente difieren de acuerdo a lo que se muestra en la tabla 3. Por su parte, las medias de las variables formalización y especialización en el grupo tres (G3) difieren significativamente de los grupos uno y dos ( $G 1$ y $G 2$ ), pero estos dos últimos no son diferentes significativamente aunque numéricamente se observan con valores distintos según los resultados de la tabla 3.

En cuanto a la variable centralización, se aprecia que el valor de la media del grupo uno (G1) de 2,93 es significativamente menor a la media de los grupos dos (G2) de 4,13 y tres (G3) de 4,14 , pero la de estos dos últimos grupos no son significativamente diferentes entre sí, a pesar de que la media de G3 es mayor cuantitativamente a la de G2 -ver tabla 3-.
Estos resultados llevan a asignarle valores a la variable centralización de bajo en G1 y de alto en G2 y G3.

Con respecto a la variable formalización, las medias de los grupos uno (G1) de 4,21 y dos (G2) de 4,34 no son diferente significativamente, pero ambas lo son respecto a la del grupo tres (G3) de 3,01; por lo tanto, se le asignan un valor cualitativo de alto en G1 y G2 y de medio-bajo en G3. Por su parte, para la variable especialización, al igual que la formalización, el valor de las medias de los grupos uno (G1) de 3,65 y dos (G2) de 3,98 no son diferente significativamente, pero ambas lo son en relación a la del grupo tres (G3) de 3,27, en consecuencia, se le asignan un valor cualitativo de medioalto en $\mathrm{G} 1$ y G2 y de medio-bajo en G3.

En la tabla 5, se muestra la distribución de las empresas agrupadas según las formas de organización con las medias correspondiente a cada variable de diseño organizativo. Estos resultados permiten responder a las hipótesis $\mathrm{H} 1$.a, H2.a y H2.b, formuladas en la sección 4.

Tabla 5

Formas de Organización en las Medianas Empresas del Estado Lara, Venezuela

\begin{tabular}{cccc}
\hline $\begin{array}{c}\text { Variables de } \\
\text { Diseño }\end{array}$ & Grupo 1 $(\mathrm{NE}=10,16,39 \%)$ & Grupo 2 (NE=29, 47,54\%) & Grupo3 $(\mathrm{NE}=22,36,07 \%)$ \\
Organizativo & Teoría Burocrática & Teoría Orgánica & Teoría Burocrática \\
& Burocrática Compleja & Forma Mecánica & Burocrática Simple \\
Centralización & Bajo $(2,9)$ & $(\mathrm{H} 1 . \mathrm{H})$ & Alto $)$ \\
\hline Formalización & Alto $(4,21)$ & Alto $(4,13)$ & Medio-Bajo $(3,01)$ \\
\hline Especialización & Medio-alto $(3,65)$ & Alto $(4,34)$ & Medio-Bajo $(3,27)$ \\
\hline
\end{tabular}

Nota: El sombreado indica que las variables en cada grupo no difieren significativamente.

Fuente: Elaboración propia.

La clasificación de acuerdo al tipo de forma de organización en la tabla 5 , se obtiene de la siguiente manera: (a)
El grupo uno de empresas (G1), posee características que tienden a definirla como forma burocrática compleja 
puesto que mantiene niveles bajos de centralización, es decir, una tendencia a la delegación -descentralización- en la toma de decisiones acompañado de una tendencia a elevados niveles de formalización y de especialización del trabajo, este resultado ratifica la hipótesis H2.b. (b) El grupo dos de empresas (G2), se identifica con la forma de organización mecánica ya que las tres variables de diseño mantienen una tendencia a valores elevados, y se corresponde a lo afirmado en la hipótesis H1.a. (c) El grupo tres (G3) de empresas, posee características de una burocracia simple puesto que tiene altos niveles de centralización acompañado de una tendencia a valores bajos de formalización y especialización, corroborándose el planteamiento de la hipótesis H2.a.

Adicionalmente, se observa en los resultados de la tabla 5 que la mayor cantidad de empresas, el $83,61 \%$, se concentran en los tipos de formas de organización mecánica y burocracia simple. Esto permite interpretar que una de las características más resaltantes de la población de medianas empresas del Estado Lara es que tiende a tomar decisiones de manera centralizada; es decir, en los niveles superiores de su jerarquía, con lo cual se entiende un tipo de gerencia que concentra en su actividad la toma de decisiones importante para la organización.

Con respecto a la hipótesis $\mathrm{H} 3$, formulada a partir de la teoría burocrática, en cuanto a que existe una correlación negativa entre la formalización y la especialización con respecto a la centralización, se comprueba de acuerdo a los resultados presentados en la tabla 6. Esto, debido a que las correlaciones positivas o negativas, según el caso, son significativas a nivel 0,01 . Se observa así una tendencia a la descentralización y a niveles más elevados de formalizaciónespecialización en la burocracia compleja (G1), y una tendencia en la burocracia simple (G3) a mayores niveles de centralización y menores de formalización-especialización.

Tabla 6

Correlaciones entre las Variables de Diseño en las Formas Burocráticas

\begin{tabular}{cccc}
\hline Variables & Centralización & Formalización & Especialización \\
\hline Centralización & 1 &,$- 703^{* *}$ &,,$- 315^{* *}$ \\
\hline Formalización &,$- 703^{* *}$ & 1 &, $406^{* *}$ \\
\hline Especialización &,$- 315^{* *}$ &, $406^{* *}$ & 1 \\
\hline
\end{tabular}

** La correlación es significativa al nivel 0,01 (bilateral).

Fuente: Elaboración propia.

En los resultados obtenidos, no se vio reflejado en el estudio empírico el tipo de forma orgánica, por lo tanto no pudo ser verificada la hipótesis H1.b. Esto se debe al hecho de que un $83,61 \%$ de las empresas de la muestra son burocracias simples y formas mecánicas, que tienden a tener elevados valores de centralización. Pero en el caso de presentar una tendencia 
Características de diseño organizativo de las medianas empresas del estado Lara...

Gerardo J. Zapata Rotundo, Luis E. Sigala Paparella, María Teresa Canet Giner

a la descentralización, los niveles de formalización y especialización se incrementan tal como se observa en los resultados, y que se corresponde al tipo de burocracia compleja. Es decir, empresas, que junto a las formas mecánicas, asignan un mayor número de empleados a roles especializados e implementan una mayor estandarización en los procesos de trabajo y en las tareas de cada puesto trabajo codificados y registrados en manuales y documentos de operación, características estas que no se corresponden precisamente a las formas de organización orgánicas. Con lo cual, la actuación de los gerentes y directivos en el proceso de supervisión y control en las medianas empresas objeto de estudio, alcanza un rol relevante, cuestión que coincide con los planteamientos teóricos para este tipo de organizaciones.

\section{Conclusiones}

Los resultados permitieron cumplir con el objetivo de la investigación, puesto que se han aportado evidencias empíricas sobre un tema central en la teoría de la organización, como lo es el estudio de las características de diseño organizativo de las empresas en los niveles de análisis micro, meso y macro organizativo.

Se identificaron y caracterizaron los tipos de formas organizativas y el nivel alcanzado por las variables centralización, formalización $y$ especialización dentro del marco de la teoría orgánica y la teoría burocrática, respondiendo al objetivo de carácter descriptivo y preliminar planteado inicialmente en este trabajo. Por otra parte, con el trabajo empírico se ha hecho operativa la relación entre esas variables de diseño, los tipos de estructuras organizativas y las formas de organización mecánica y burocrática que adoptan las medianas empresas del Estado Lara, Venezuela.

Destaca por su alta frecuencia la estructura organizativa de tipo funcional, propia de organizaciones que comercializan o elaboran uno o pocos productos, o en caso de que se involucre con una gran variedad de ellos, estos deben estar muy relacionados tecnológica y comercialmente. Asimismo, en ellas se establecen formas de agrupación de unidades organizativas de acuerdo con las especialidades o actividades homogéneas que en ellas se realizan, permitiéndoles alcanzar economías de escala puesto que se aprovecha una base de recursos $y$ conocimientos colectivos que evita la repetición de unidades con las mismas funciones.

De igual modo, la investigación permitió identificar que la forma de organización de las medianas empresas objeto estudio van desde la burocracia compleja, en un menor porcentaje (16,39\%: descentralizadas y formalizadas), hasta la burocracia simple $(47,54 \%$ : centralizadas y menos formalizadas) y la forma mecánica (36,07\%: centralizadas y formalizadas). Esto quiere decir que en general tienden a ser centralizadas y formalizadas en su funcionamiento, por lo que concentran la autoridad para la toma decisiones estratégicas, y en buena medida las operativas, en los niveles superiores de la pirámide empresarial, y que se corresponden con "aquellas decisiones ambiguas, arriesgadas $y$ que se extienden a través de múltiples dimensiones funcionales y ambientales que requieren de un liderazgo fuerte" (Liang et al., 2010). Por su parte, 
aquellas decisiones muy particulares o específicas para cada puesto de trabajo vinculadas con las actividades diarias, rutinarias y a corto plazo, se dejan para los niveles más operativos.

$$
\text { Asimismo, establecen }
$$
mecanismos formales para controlar y supervisar el comportamiento y actuaciones de sus miembros, cuando se enfrentan a entornos muy complejos y con elevados niveles de incertidumbre, o cuando su funcionamiento depende, en gran medida, de otras organizaciones o de situaciones que están fuera de su control, como ocurre actualmente en el entorno empresarial venezolano. Por ser formalizadas, tienen una tendencia a registrar y codificar en documentos o manuales de organización las formas de comportamientos y los métodos trabajo de sus empleados, y otros aspectos organizativos vinculados con sus objetivos, misión, visión y mecanismos de coordinación y control; es decir, se establece un marco claro para la centralización en la toma de decisiones y la formalización organizativa como mecanismos de control y coordinación del trabajo, y en general, de las actividades llevadas a cabo en la organización.

Es importante señalar que aunque no se obtuvo evidencia empírica sobre las características estructurales de la forma orgánica, quizás se corresponde principalmente a empresas de tamaño pequeño donde los mecanismos de comunicación tienden a ser más bien laterales y personales, y la formalización toma un carácter menos codificado y poco relevante como instrumento administrativo para controlar, supervisar y coordinar a los empleados en sus puestos de trabajo, a diferencia de lo que ocurre en las medianas o grandes organizaciones. En estas últimas, se puede presentar un mayor distanciamiento entre los centros de trabajo -unidades organizativasubicados en los niveles medios y bajos de las jerarquía y la dirección central de la organización, que genera la necesidad de implementar mecanismos indirectos de control y supervisión a través de la formalización, características estas que no se corresponden con las formas orgánicas. En todo caso, es una circunstancia que hay que evaluar en posteriores estudios donde se involucren otros tipos y tamaños de organizaciones.

De cualquier modo, conocer las características de las empresas venezolanas facilitará detectar las debilidades y fortalezas de estas organizaciones en términos estructurales, lo cual puede llevar al diseño de propuestas que ayuden a flexibilizar, en la medida de lo posible y dependiendo de las características de la industria, las estructuras de estas empresas que permitan gestionar adecuadamente los nuevos requerimientos que la tecnología exige; es decir, para gestionar conocimiento, introducir nuevas tecnologías y avanzar en procesos de innovación que tienden a poner énfasis en el factor humano, en su talento, y, por tanto, descentralizar las estructuras manteniendo ciertos niveles de formalización como mecanismo de control y orientación del trabajo, o de introducir el trabajo en equipo. Todo con el propósito de aprovechar en la mejor medida posible los recursos y las capacidades internas que poseen, de esta manera tratar de alcanzar ventajas competitivas sostenibles que le permitan desarrollarse y subsistir en un entorno cada vez más complejo y competitivo.

$\begin{array}{ccc}\text { El presenta estudio posee } & \text { pofieren }\end{array}$ fundamentalmente al tamaño de la muestra y a la subjetividad inherente 
Características de diseño organizativo de las medianas empresas del estado Lara... Gerardo J. Zapata Rotundo, Luis E. Sigala Paparella, María Teresa Canet Giner

en las respuestas que puede haber en este tipo de estudio, ya que depende del sujeto que responde a la encuesta y a las circunstancias en la cual lo hace.

En cuanto a futuras investigaciones, es necesario seguir aplicando otras técnicas estadísticas y procedimientos metodológicos en poblaciones de empresas de rango de tamaños diferentes con el propósito continuar perfeccionando su caracterización desde el punto de vista de su diseño organizativo, y así obtener nuevas y mejores evidencias empíricas que fortalezcan los fundamentos teóricos que sustenten modelos organizacionales cada vez más elaborados y cercanos a la realidad empresarial. En este sentido, esta investigación se presenta como una guía orientadora desde el punto de vista metodológico para el estudio de las características de diseño organizativo de las empresas, independientemente de su tamaño, naturaleza o ámbito de acción.

Por otra parte, el estudio de la pequeña empresa y de cómo su carácter orgánico y su mayor flexibilidad pueden contribuir al dinamismo e innovación de las empresas y los mercados, es un reto para futuros trabajos.

\section{Referencias bibliográficas}

Akingbola, Kunle (2013), Contingency, Fit and Flexibility of HRM in Nonprofit Organizations. Employee Relations, Vol. 25, No. 5, pp. 479-49.

Aragón, Antonio y Rubio, Alicia (2009), ¿Cómo Compiten las Empresas Industriales? Un Estudio sobre las PyMEs Españolas. Economía industrial, No. 373, pp. 87-99.

Aristy Escuder, Jaime (2012), Determinantes del Éxito de las pymes en la República Dominicana. Ciencia y Sociedad, Vol. 37, No. 4, pp. 497-528.
Arteaga, Jesús; Fernández; Rubén y Clavel, Mónica (2014), Las Pymes Españolas en Latinoamérica: Barreras a la Exportación. Ad-minister, No. 24, pp. 53-78.

Biggiero, Lucio y Laise, Domenico (2003), Comparing and Choosing Organizational Structures: A Multicriteria Methodology. Human Systems Management, Vol. 22, No. 4, pp. 185-195.

Bodewes, Wynand (2002), Formalization and Innovation Revisited. European Journal of Innovation Management, Vol. 5, No. 4, pp.214-223.

Bonner, Joseph, Ruekert, Robert y Walker, Orville (2002), Upper Management Control of New Product Development Projects and Project Performance. Journal of Product Innovation Management, Vol. 19, No. 3, pp. 233245.

Boonpattarakan, Apicha (2012), Model of Thai Small and Medium Sized Enterprises' Organizational Capabilities: Review and Verification. Journal of Management Research, Vol. 4, No.3, pp. 15-42.

Burns Tom y Stalker G.M (1961), The Management of Innovation. Ediciones Tavistock Publications.

Chen, Chung-Jen; Huang, Jing-Wen y Huang, Yung-Chang (2010), Knowledge Management and Innovativeness: The Role of Organizational Climate and Structure. International Journal of Manpower, Vol. 31 No. 8, pp. 848870.

Child, John (1973), Predicting and Understanding Organization Structure. Administrative Science Quarterly, Vol. 18, No. 2, pp. 168185.

Child, John (2008), Organization. Contemporary Principles and Practice. Blackwell Publishing, Oxford, UK.

Claver-Cortés, Enrique; Pertusa-Ortega, Eva y Molina-Azorín, José (2006), Características de las Estructuras Mecánica y Orgánicas a Partir de las Principales Dimensiones Estructurales. Investigaciones Europeas de Dirección y Economía de la Empresa, Vol. 12, No. 2, pp. 187-204 
Claver-Cortés, Enrique; Pertusa-Ortega, Eva y Molina-Azorín, José (2012), Characteristics of Organizational Structure Relating to Hybrid Competitive Strategy: Implications for Performance. Journal of Business Research, Vol. 65, pp. 993-1002.

Courtright, John; Fairhurst, Gail y Rogers, Edna (1989), Interaction Patterns. In: Organic and Mechanistic Systems. Academy of Management Journal, Vol. 32, No. 4, USA, pp. 773-802.

Cronbach, Lee Joseph (1951), Coefficient Alpha and the Internal Structure of Test. Psychometrika, Vol. 16, pp. 297-334.

Csaszar, Felipe (2012), Organizational Structure as a Determinant of Performance: Evidence From Mutual Funds. Strategic Management Journal, Vol. 33, pp. 611-632.

Daft, Richard (2000). Teoría y Diseño de la Organización. México: International Thomson.

Delić, Adisa y Ahmetović, Emir (2013), Characteristics of Organizational Structure of Bosnian and Herzegovinian Companies. Journal of Economics and Business, Vol. $\mathrm{XI}$, No. 2, pp. 31-43.

Demirci; Emre (2013), In Pursuit of Corporate Entrepreneurship: How Employees Perceive the Role of Formalization and Centralization. Journal of Management Research, Vol. 5, No. 3, pp. 115-133.

Donaldson, Lex (2001), The Contingency Theory of Organizations. London, Sage Publications.

Fiedler, Marina y Welpe, Isabell (2010), How do Organizations Remember?. The Influence of Organizational Structure on Organizational Memory. Organization Studies, Vol. 31, No. 4, pp. 381-407.

Foss, Nicolai; Lyngsie, Jacob y Zahra, Shaker (2015), Organizational Design Correlates of Entrepreneurship: The Roles of Decentralization and Formalization for Opportunity Discovery and Realization. Strategic Organization, Vol. 13, No. 1, pp. 3260.
Gallardo, Luis y Avilés, Ezequiel (2014), Estructura de Capital y Riesgo Financiero: Evidencia Empírica en Pymes Hoteleras. Global Conference on Business \& Finance Proceedings, Vol. 9, No. 2, pp. 12751284.

García, María Concepción (2003), La Medición de la Estructura Organizativa. Revista Europea de Dirección y Economía de la Empresa, Vol. 12, No. 3, pp. 1163-176.

Gibson, James; Ivancevich John, Donnelly, James y Konopaske, Robert. (2011) Organizaciones. Comportamiento, Estructura y Procesos. McGrawHill, $13^{a}$ Edición.

Glock, Christoph y Broens, Michael (2013), Size and Structure in the Purchasing Function: Evidence from German Municipalities. Journal of Public Procurement, Vol. 13, No. 1, pp. 1-38.

González, Tomás; Huguet, Ana y Cruz, Sonia (2012), Organizational Technology as a Mediating Variable in CentralizationFormalization Fit. Management Decision, Vol. 50, No. 9, pp. 15271548.

Grinyer, Peter y Ardekani-Yasay, Masoud (1981), Strategy, Structure, Size and Bureaucracy. Academy of Management Journal, Vol. 24, No. 3, pp. 471-486.

Hair, Joseph; Anderson, Rolph; Tatham, Ronald y Black, William (1999), Análisis Multivariante. Madrid: Prentice Hall.

Hall, Richard (1996), Organizations, Structures, Processes and Outcomes. Prentice Hall International, Inc. Nueva York.

Hwang, Eun Jin y Norton, Marjorie (2014), Environmental Uncertainty, Market-Orientation Strategy, and Organizational Structure in China's Apparel Retail Stores. Advances in Business-Related Scientific Research Journal, Vol. 5, No. 1, pp. 1-12.

Hempel, Paul; Zhang, Zhi-xue y Han, Yulan (2012), Team Empowerment and the Organizational Context: Decentralization and the Contrasting Effects of Formalization. Journal of 
Características de diseño organizativo de las medianas empresas del estado Lara... Gerardo J. Zapata Rotundo, Luis E. Sigala Paparella, María Teresa Canet Giner

Management, Vol. 38 No. 2, pp. 475501.

Hodge, Billy; Anthony, William y Gales, Lawrence (1998), Teoría de la Organización. Un Enfoque Estratégico. Prentice Hall, Madrid.

Instituto Nacional de Estadistica (2010), IV Censo Económico (2007-2008). http://www.ine.gov.ve/documentos/ Economia/IVCensoEconomico/pdf/ InformelVCE.pdf.

Janićijević, Nebojša (2013), The Mutual Impact of Organizational Culture and Structure. Economic Annals, Vol. LVIII, No. 198, pp. 35-60.

Kloviene, Lina (2013), Improvement of the Performance Measurement System According to Business Environment. Economics and Management, Vol. 18, No. 1, pp. 17-25.

Liang, Xin; Achidi, Ndofor Hermann; Priem, Richard y Picken, Joseph (2010), Top Management Team Communication Networks, Environmental Uncertainty, and Organizational Performance: A Contingency View. Journal of Managerial Issues, Vol. 22, No. 4, pp. 436-455.

Liao, Yao-Sheng (2007), The Effects of Knowledge Management Strategy and Organization Structure on Innovation. International Journal of Management, Vol. 24, No. 1, pp. 5360.

López, Liliana; Tricás, Jesús y Toledano, Rubén (2012), Principales Prácticas de Recursos Humanos de las PyMEs Industriales Exitosas. Universidad \& Empresa, Vol. 23, pp. 19-43.

Mahmood, Sharareh; Musriyama, Andira y Hosseinipour, Seyed (2013), Relationship Between Organizational Structure and Knowledge Sharing Culture. Interdisciplinary Journal of Contemporary Research in Business, Vol. 5, No.2, pp.518-524.

Mao, Hsiao-Yen; Chen, Chien-yu y Hsieh, Ting-Hua (2009), The Relationship Between Bureaucracy and Workplace Friendship. Social Behavior and Personality, Vol. 30, No. 2, pp. 255-266.

Mansfield, Roger (1973), Bureaucracy and Centralization: An Examination of Organizational Structure.
Administrative Science Quarterly, Vol. 18, No. 4, pp. 477-488.

Marín-Idárraga, Diego y Cuartas-Marín, Juan (2013), Structural Co-alignment Influence on SMEs Performance. International Journal of Business \& Management, Vol. 8, No. 22, pp. 76-91.

Marín-Idárraga, Diego y Losada-Campos, Ángela (2015), Estructura Organizacional y Relaciones Inter-organizacionales: Análisis en Instituciones Prestadoras de Servicios de Salud Pública de Colombia. Estudios Gerenciales, Vol. 31, No. 134, pp.88-89.

Miller, Danny (1987), Strategy Making and Structure: Analysis and Implications for Performance. Academy of Management Journal, Vol. 30, No. 1, pp. 7-32.

Milne, Robert (1970), Mechanistic and Organic Models of Public Administration in Developing Countries. Administrative Science Quarterly, Vol. 15, No. 1, pp. 57-67.

Mintzberg, Henry (1999), La Estructuración de las Organizaciones. Editorial Ariel, S.A. Barcelona. Sexta Reimpresión en Castellano.

Nasurdin, Aizzat Mohd. Romayah y Beng, Yeoh (2006), Organizational Structure and Organizational Climate as Potential Predictors of Job Stress: Evidence from Malaysia. International Journal of Commerce and Management, Vol. 16, No. 2, pp. 116-129.

Narváez, Mercy y Fernández, Gladys(2013), Redes de Cooperación Empresarial: Relaciones e Interacciones para Promover Desarrollo Turístico Local. Revista Venezolana de Gerencia (RVG), Vol. 18, No. 61, pp. 121-137.

Nohria, Nitin y Ghoshal, Sumantra (1994), Differentiated Fit and Share Values: Alternatives for Managing Headquarters-Subsidiary Relations. Strategic Management Journal, Vol. 15, pp. 491-502.

Nonaka, Ikujiro y Takeuchi Hirotaka (1995), The Knowledge-Creating Company. New York: Oxford University Press.

Nunnally, Jum y Bernstein, Ira (1995), Teoría Psicométrica. McGraw-Hill/ Interamericana de México, S.A. Tercera edición. 
Pelechano, Eva; González, Rocío y García, Fernando (2015), La Configuración de la Red Interempresarial y el Desempeño Innovador: Una Relación Moderada por la Capacidad de Asimilación de Conocimiento. XXV Congreso Nacional de ACEDE, Jaén, España.

Pertusa-Ortega, Eva; Molina-Azorín, Jose y Claver-Cortés, Enrique (2010), Competitive Strategy, Structure and Firm Performance. A Comparison of The Resource-Based View and the Contingency Approach. Management Decision, Vol. 48 , No. 8, pp. 12821303.

Prakash, Yamini y Gupta, Meenakshi (2008), Exploring the Relationship Between Organization Structure and Perceived Innovation in the Manufacturing Sector of India. Singapore Management Review, Vol. 30, No. 1, pp. 55-76.

Pleshko, Larry (2007), Strategic Orientation, Organisational Structure, and the Associated Effects on Performance. Journal of Financial Services Marketing, Vol. 12, No. 1, pp. 53-64.

Pugh, Derek y Hickson, David (1976), Organizational Structure in Its Context: The Aston Programmed I. USA: Saxon House Studies.

Puranam, Phanish; Alexy, Oliver; Reitzig, Markus (2014), What's "New" About New Forms Of Organizing?. Academy of Management Review, Vol. 39 No. 2, pp. 162-180.

Reichmann, Steffen y Rohlfing-Bastian, Steffen (2014), Decentralized Task Assignment and Centralized Contracting: On the Optimal Allocation of Authority. Journal of Management Accounting Research, Vol. 26, No.1, pp. 33-55.

Ramezan, Majid (2011), Intellectual Capital and Organizational Organic Structure in Knowledge Society: How are These Concepts Related? International Journal of Information Management, Vol. 31, No. 1, pp. 8895.

Russell, Robert y Russell, Craig (1992), An Examination of the Effects of Organizational Norms, Organizational Structure, and Environmental Uncertainty on Entrepreneurial
Strategy. Journal of Management, Vol. 18, No. 4, pp. 639-656.

Salazar Castillo, José Manuel (2005), La estructura organizativa en la empresa actual. El caso Inditex. Boletín Económico de ICE, No 2850, pp. 7-19.

Salim, Alawamleh Hamdan y Abdalraheem Kloub Mohammad (2013), Impact of Organizational Structure on Knowledge Management in the Jordanian Insurance Companies: From the Perspective of the Supervisory Leadership. International Journal of Business and Social Science, Vol. 4 No. 11, pp. 82-95.

Simon, Cadez (2007), A configuration Form of Fit in Management Accounting Contingency Theory. An empirical investigation. The Business Review, Cambridge, Hollywood, Vol. 7, No. 2, pp. 220-227.

Tiller, Stephen (2012), Organizational Structure and Management Systems. Leadership \& Management in Engineering, Vol. 12, No. 1, pp. 2023.

Tyulkova, Natalia (2014), A Flexible Organizational Structure as a way of Knowledge Management in SMEs. Proceedings of the International Conference on Intellectual Capital, Knowledge Management \& Organizational Learning, pp. 549557.

Volberda, Henk; Weerdt, Niels Van Der; Verwaal, Ernst; Stienstra, Marten y Verdu, Antonio (2012), Contingency Fit, Institutional Fit, and Firm Performance: A Meta Fit Approach to Organization-Environment Relationships. Organization Science, Vol. 23, No. 4, pp. 10401054.

Weber, Max (1947), The Theory of Social and Economic Organization. New York: Free Press.

Wynen, Jan; Verhoest, Koen y Rübecksen, Kristin (2014), Decentralization in Public Sector Organizations. Do Organizational Autonomy and Result Control Lead to Decentralization Toward Lower Hierarchical Levels?. Public Performance \& Management Review, Vol. 37, No. 3, pp. 496-520. 
Características de diseño organizativo de las medianas empresas del estado Lara...

Gerardo J. Zapata Rotundo, Luis E. Sigala Paparella, María Teresa Canet Giner

Zainun, Tuan; Mat, Tuan y Malcolm, Smith (2014), The Impact of Changes in Environment and AMT on Management Accounting Practices and Organizational Strategy, Structure and Performance. Journal of Applied Management Accounting Research, Vol. 12, No. 1, pp. 55-82.

Zanzi, Alberto (1987), How Organic is Your Organization? Determinants of Organic/Mechanistic Tendencies in a Public Accounting Firm. Journal of Management Studies, Vol. 24, No. 2, pp. 125-142.

Zapata Rotundo, Gerardo y Canet Giner, María Teresa (2012), Tipos de Trabajo y la Formación de la Especialización de Tareas en la Organización. Revista de Ciencias Sociales (RCS), Vol. XVIII, No. 1, pp. 58-73.

Zapata Rotundo, Gerardo y Mirabal, Alberto (2013), Contingencia, Voluntarismo y Strategic Choice: Un Análisis Teórico de sus Enfoques sobre el Entorno y el Diseño de la Organización. Revista Universidad y Empresa, Vol. 15, No. 34, pp. 119-139.

Zapata Rotundo, Gerardo y Hernández, Aymara (2014), La Empresa: Diseño, Estructuras y Formas Organizativas. Segunda Edición. Venezuela: Editorial Horizonte, C.A. 


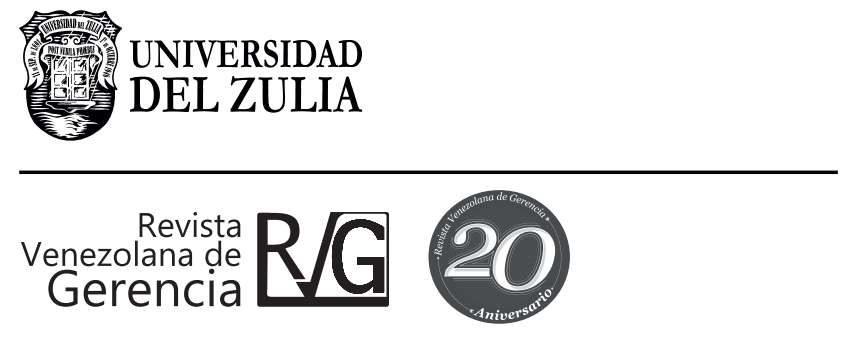

Año 21, No. 74

Esta revista fue editada en formato digital y publicada en junio del 2016, por la Universidad del Zulia, Vicerrectorado Académico, Serbiluz - Fondo editorial, Maracaibo -Venezuela. 